\title{
Conhecimento dos enfermeiros sobre o Serviço de Atenção às Mulheres Vítimas de Violência Sexual
}

\author{
Knowledge of nurses about the Service for Assistance to Women Victims of Sexual Violence
}

Conocimiento de los enfermeros sobre el Servicio de Atención as las Mujeres Víctimas de Violencia Sexual

\section{Claudete Ferreira de Souza Monteiro', Sheila Coelho Ramalho Vasconcelos Morais', Maria Tamires Alves Ferreira', Rodolfo Xavier da Costa Carvalho', Mary Ângela de Oliveira Canuto', Isabel Cristina Cavalcante Carvalho Moreira' \\ 'Universidade Federal do Piauí, Grupo de Estudo sobre Saúde, Gênero e Vioência. Teresina, PI}

\section{RESUMO}

Estudo quantitativo para levantar o conhecimento dos enfermeiros sobre o Serviço de Atenção às Mulheres Vítimas de Violência Sexual (SAMVVIS), de uma maternidade pública-Teresina, PI. A coleta deu-se em fevereiro e março de 2008, com 6I enfermeiras(o). Os resultados apontam Que a maioria conhece o serviço $(90,14 \%)$, mas os percentuais afirmativos vão decrescendo à medida Que respondem como funcionam $(80,33 \%)$, Quais seus objetivos $(72,13 \%)$, o papel do enfermeiro $(44,26 \%)$ e a proposta de organização (40,98\%). Conclui-se da necessidade de divulgação do serviço, notadamente nos aspectos dos objetivos, da proposta de organização e do papel do enfermeiro, bem como da política nacional de atenção às mulheres vítimas de violência sexual.

Desscritores: Violência sexual; Conhecimento; Enfermagem.

\section{ABSTRACT}

This Quantitative study is to collect data of the knowledge of nurses about the Service for Assistance to women victims of sexual violence (SAMVVIS), in a public maternity hospital in Teresina, PI. Data was collected in February and March of 2008 from $6 \mathrm{I}$ nurses. The results show that the majority are aware of the service (90.14\%) but the affirmative percentage decreases when asked about how it functions $(80.33 \%$, what its objectives are $(72.13 \%)$, the role of the nurse $(44.26 \%)$ and the proposal of the organization $(40.98 \%)$. It follows that there is a need for the diffusion of the service, especially in the aspect of its objectives, the proposal of the organization and the role of the nurse along with national political attention for the women who are victims of sexual violence.

Descriptores: Sexual violence; Knowledge; Nursing.

\section{RESUMEN}

Estudio cuantitativo para verificar el conocimento de los enfermeros sobre el Servicio de Atención a las Mujeres Víctimas de Violencia Sexual (SAMVVIS), de una maternidad pública-Teresina, PI. La recolección se dio en febrero y marzo de 2008, con 6I enfermeras(os). Los resultados apuntan Que la mayoría conoce el servicio $(90,14 \%)$, pero los porcentajes afirmativos van disminuyendo a la medida Que responden cómo funcionan $(80,33 \%)$, cuáles sus objetivos $(72,13 \%)$, el papel del enfermero $(44,26 \%)$ y la propuesta de organización (40,98\%). Se concluye la necesidad de divulgación del servicio, especialmente en los aspectos de los objetivos, de la propuesta de organización y del papel del enfermero, así como de la política nacional de atención a las mujeres víctimas de violencia sexual.

Descriptores: Violencia sexual; Conocimento; Enfermería. 


\section{INTRODUÇÃO}

A violência contra a mulher entendida como conduta Que se baseia no gênero, resultando em morte ou que provoque dano ou sofrimento físico, sexual ou psicológico à mulher, é verificada tanto no espaço privado como público. Dentre as várias formas, destacase a violência sexual por apresentar particularidades importantes Que merecem ser tratadas com relevância, já Que, na maioria das vezes, se encontra seguida de outras expressões, como alterações físicas, reprodutivas, sociais e psicológicas, produzindo graves danos à saúde das mulheres ${ }^{(1,2)}$.

Esse tipo de violência não decorre do desejo sexual e amoroso, e sim de uma forma de provar a relação de domínio existente entre homens e mulheres, as Quais são consideradas meramente objetos desprovidos de independência Quanto as suas vidas e de respeito como membros participantes de uma sociedade livre ${ }^{(3)}$.

As conseqüências de origem imediata podem manifestar-se como infecções do trato reprodutivo, como DSTs/AIDS, e a possibilidade de uma gravidez indesejada. A mulher pode experimentar uma série de sentimentos negativos, como o medo da morte, solidão, vergonha e até uma sensação de culpa, Que acabam por evoluir para alguns tipos de transtornos psíQuicos, como fobia, pânico e depressão. Deve-se também levar em conta alguns aspectos sociais, como abandono da escola, emprego e do lar, separação, prostituição, entre outros ${ }^{(4,5)}$.

Estima-se Que, por ano, 12 milhões de mulheres sejam vítimas de violência sexual em todo o mundo. Apesar de representar um fenômeno universal e destituído de fronteiras, Que não faz distinção entre classes, cor ou etnia, a verdadeira incidência dos crimes sexuais contra a mulher é desconhecida. Isso se deve a subnotificação e o sub-registro dos casos, uma vez Que a mulher tem medo de se expor, envergonha-se do acontecido e teme represálias do agressor, Que no geral são parentes, pessoas próximas ou conhecidas ${ }^{(6,7)}$.

Apesar de a violência sexual ocorrer em todas as faixas etárias, a maioria dos registros aponta para a predominância desses crimes entre adolescentes e adultas jovens ${ }^{(6)}$.

No Brasil, ainda são poucos os dados. Estima-se que os registros das delegacias correspondam apenas a 10 a 20\% dos casos Que realmente acontecem. Entretanto, apesar da escassez de dados, os números observados mostram uma realidade assustadora, Que é a elevada incidência e prevalência dos crimes sexuais e a repercussão sobre a saúde da mulher vitimada, mostrando a necessidade de estudos Quantitativos e Qualitativos para melhor entender e dimensionar o problema ${ }^{(8)}$.

Para entender o fenômeno violência sexual e atuar sobre suas conseqüências, faz-se necessário a participação de diversos profissionais e segmentos, pois não se trata de uma violência unilateral e sim multifacetada Que impõe uma série de desafios à intervenção pública ${ }^{(9)}$.

Diante disso, nos últimos anos, o atendimento às mulheres vítimas de violência sexual tem recebido maior atenção de diferentes setores da sociedade, com sua incorporação no rol das ações dos serviços de saúde, resultando na formação de recursos humanos e na criação de serviços Que atendem mulheres vítimas de violência sexual ${ }^{(10)}$.

No Que diz respeito às ações de atenção à saúde das mulheres, em 1999, o Ministério da Saúde publica a Norma Técnica de
Prevenção e Tratamento dos Agravos Decorrentes de Violência Sexual Contra Mulheres e Adolescentes, com o objetivo de apoiar municípios e estados na promoção da saúde, prevenção e tratamento do problema. A partir de então, o Ministério vem capacitando profissionais e equipando os serviços para diagnosticar os casos de violência, promover assistência adeQuada, desenvolver e ampliar serviços especializados e redes de referência Que permitam melhor acesso das mulheres a estes equipamentos ${ }^{(1)}$.

No estado do Piauí, a partir de março de 2002, foi criado em Teresina o Projeto Maria-Maria, em uma maternidade pública, para atender a mulher vítima de violência sexual. A partir de 2004, o Projeto Maria-Maria passou a se chamar SAMVVIS (Serviço de Atenção às Mulheres Vítimas de Violência Sexual). Esse serviço tem como objetivo viabilizar o acesso imediato das mulheres vitimizadas às medidas de prevenção das DSTs/AIDS, gravidez e outros agravos físicos e psicológicos ${ }^{(12)}$.

O SAMVIS tem como eixo norteador a Norma Técnica de Prevenção e Tratamento dos Agravos Resultantes da Violência contra Mulheres e Adolescentes, do Ministério da Saúde, no Qual propõe Que as ações de saúde sejam desempenhadas por uma equipe multiprofissional, incluindo médicos(as), psicólogos(as), enfermeiros(as), assistentes sociais, técnicos de enfermagem e outros, se necessário.

Segundo os dados fornecidos pela Secretaria de Estado da Saúde do Piauí, através da Coordenação de Atenção à Saúde da Mulher, o SAMVVIS registrou 688 casos de atendimento de mulheres vítimas de violência sexual, no período de 2000 a $2005^{(13)}$.

$\mathrm{Na}$ proposta de organização do SAMVVIS, a equipe de enfermagem é responsável pela administração de medicamentos, vacinas e do agendamento das doses subseqüentes, orientações na anticoncepção de emergência, Quimioprofilaxia para infecção pelo vírus HIV e outras doenças sexualmente transmissíveis, avaliação das necessidades básicas afetadas, realização do teste rápido para o HIV, agendamento do retorno para avaliação ginecológica e repetição dos exames laboratoriais, como VDRL, HIV, Hepatite B e C, Hemograma e Transaminases ${ }^{(12)}$.

Entretanto, observa-se Que não há a participação direta da(o) enfermeira(o) na equipe do SAMVVIS, e, conseQüentemente, não está implantado a consulta de enfermagem para de fato dar acolhimento e prosseguimento às orientações necessárias a essas mulheres, o Que nos levou a Questionar sobre se esses profissionais detêm conhecimento sobre a existência desse serviço, da proposta e do seu papel dentro da equipe.

Tomando por base esses Questionamentos, e da possibilidade de trabalharmos junto a dois projetos, do Programa de PósGraduação Mestrado em Enfermagem e por serem poucas as pesquisas locais acerca da temática violência contra a mulher, realizou-se este estudo com o objetivo de levantar o conhecimento das(os) enfermeiras(os) sobre o Serviço de Atenção às Mulheres Vítimas de Violência Sexual (SAMVVIS).

\section{METODOLOGIA}

Trata-se de uma peseuisa com abordagem Quantitativa, realizada em uma maternidade pública de Teresina (PI), onde funciona o SAMVVIS. O período de coleta dos dados deu-se nos meses de fevereiro e março de 2008 por meio de um Questionário, contendo 
perguntas abertas e fechadas, buscando abranger a multidimensionalidade da problemática.

O universo constitui-se de todos os enfermeiros $(n=64)$ Que trabalham nessa maternidade, dentre os Quais 61 participaram da pesquisa. Houve uma perda da população de 4 sujeitos, representando $6,35 \%$, dentro das margens previstas no estudo.

O estudo atendeu a todos os procedimentos éticos, como aprovação pelo Comitê de Ética da UFPI e a autorização da instituição, bem como a assinatura do Termo de Consentimento Livre e Esclarecido contendo as informações necessárias a habilitar os sujeitos em sua livre participação.

Os dados foram digitados e processados eletronicamente com a utilização do Software Epi-Info (versão 3.3.2). A análise utilizou estatísticas descritivas com percentuais apresentados em gráficos e tabelas e a discussão feita com base nos conhecimentos produzidos sobre o tema.

\section{RESULTADOS}

Dados referentes ao perfil social dos sujeitos apontam Que há maior prevalência de profissionais do sexo feminino $(96,72 \%)$, correspondendo a 59 profissionais. Essa é uma expressiva representatividade para o sexo feminino em relação ao sexo masculino $(3,28)$. Constatamos, também, Que as idades dos profissionais variam de 22 a 57 anos, com predominância para a faixa etária de 36 a 50 anos, equivalendo a 49, $18 \%$.

Analisando o perfil profissional dos(as) enfermeiros(os), percebese Que o tempo de formação mais prevalente foi de 8 a 15 anos (20 profissionais). No Que diz respeito ao tempo de trabalho na maternidade, a expressiva maioria $(63,93 \%)$ encontra-se na faixa de 1 a 6 anos de serviço.

O aprimoramento e a educação permanente são elementos essenciais para a capacitação, Que refletem positivamente na atuação profissional. Diante disso, um fator de relevância, Que foi abordado, relaciona-se a pós-graduação lato senso e stricto senso. Evidenciouse Que, dos 61 sujeitos, 59 possuem especialização, enQuanto
Que 2 possuem apenas a graduação, embora estes últimos estejam incluídos nas faixas de maior tempo de formação superior e de trabalho na referida maternidade.

$\mathrm{Na}$ tabela 1, buscou-se avaliar o conhecimento das(os) enfermeiras(os) sobre o SAMVVIS. Conforme observado, 55 sujeitos responderam Que conhecem $(90,16 \%)$ e 6 pesquisados $(9,84 \%)$ referem não conhecer o serviço e nem onde o mesmo funciona, enQuanto Que $2(3,28 \%)$ afirmam não saber Quem é a população alvo. Quando Questionados se o assunto SAMVVIS lhes despertava alguma forma de interesse, a maioria $(91,80 \%)$ se manifestou positivamente. Mas vale considerar Que $8,20 \%$ se manifestaram negativamente.

Para levantar o conhecimento das(os) enfermeiras(os) sobre o SAMVVIS nos seus aspectos referentes aos objetivos, a proposta de organização e o papel destes profissionais constataram-se na tabela I Que $44(72,13 \%)$ responderam Que têm conhecimento sobre os objetivos. Destes, $56,41 \%$ trabalham de 1 a 6 anos na maternidade, enquanto Que nessa mesma faixa de tempo de trabalho verifica-se Que $17(27,87 \%)$ responderam Que não conhece os objetivos, como também a proposta de organização, onde 36 profissionais $(59,02 \%)$ não conhecem. Dos entrevistados, 25 $(40,98 \%)$ afirmam conhecer. Ao investigar se esses profissionais sabem Qual é o papel da enfermeira na equipe, a maioria $(55,74 \%)$ respondeu Que não e somente 44,26\% afirmaram conhecer. Importa mostrar Que os profissionais Que detêm pouco conhecimento sobre estes aspectos encontram-se na faixa de trabalho de 1 a 6 anos.

Com relação à atuação das(os) enfermeiras(os) no SAMVVIS, dos 61 entrevistados, 26 responderam Que realizaram ou realizam cuidados de enfermagem, ao passo Que 35 informaram Que não. Neste sentido, buscou-se a informação se já haviam recebido algum tipo de treinamento, o Que fica evidenciado Que somente 5 responderam afirmativamente, enquanto que o maior número de respostas foi negativas $(21)$.

Para a importância da presença do enfermeiro dentro da equipe do SAMVVIS, 52 informaram Que sim, e apenas 3 responderam Que não acham importante fazerem parte dessa equipe. Ressalta-

Tabela 1. Conhecimento das(os) enfermeiras(os) de uma maternidade pública de Teresina-PI sobre o SAMVMS. Teresina(PI), fevereiro a março de 2008.

\begin{tabular}{|c|c|c|c|c|c|c|c|c|c|c|c|c|c|c|c|}
\hline \multirow{3}{*}{$\begin{array}{l}\text { Tópicos sobre } \\
\text { o SAMWIS }\end{array}$} & & \multicolumn{12}{|c|}{ Tempo de Trabalho na Unidade (em anos) } & \multirow{2}{*}{\multicolumn{2}{|c|}{ Total }} \\
\hline & & \multicolumn{2}{|c|}{0 a 1} & \multicolumn{2}{|c|}{2 a 6} & \multicolumn{2}{|c|}{7 a 11} & \multicolumn{2}{|c|}{12 a 16} & \multicolumn{2}{|c|}{17 a 21} & \multicolumn{2}{|c|}{22 a 26} & & \\
\hline & & $\mathrm{n}$ & $\%$ & $\mathrm{n}$ & $\%$ & $\mathrm{n}$ & $\%$ & $\mathrm{n}$ & $\%$ & $\mathrm{n}$ & $\%$ & $\mathrm{n}$ & $\%$ & $\mathrm{n}$ & $\%$ \\
\hline \multirow{2}{*}{ Conhecimento } & $\mathrm{S}$ & 2 & 100,0 & 33 & 84,6 & 2 & 100,0 & $\mathrm{I}$ & 100,0 & 10 & 100,0 & 7 & 100,0 & 55 & 90,2 \\
\hline & $\mathrm{N}$ & - & 0,0 & 6 & 15,4 & - & 0,0 & - & 0,0 & - & 0,0 & - & 0,0 & 6 & 9,8 \\
\hline \multirow{2}{*}{ Localização } & $S$ & 2 & 100,0 & 33 & 84,6 & 2 & 100,0 & 1 & 100,0 & 10 & 100,0 & 7 & 100,0 & 55 & 90,2 \\
\hline & $\mathrm{N}$ & - & 0,0 & 6 & 15,4 & - & 0,0 & - & 0,0 & - & 0,0 & - & 0,0 & 6 & 9,8 \\
\hline \multirow{2}{*}{ Interesse } & $S$ & 2 & 100,0 & 34 & 87,1 & 2 & 100,0 & 1 & 100,0 & 10 & 100,0 & 7 & 100,0 & 56 & 91,8 \\
\hline & $\mathrm{N}$ & - & 0,0 & 5 & 12,8 & - & 0,0 & - & 0,0 & - & 0,0 & - & 0,0 & 5 & 8,2 \\
\hline \multirow{2}{*}{ Funcionamento } & $S$ & 2 & 100,0 & 27 & 69,3 & 2 & 100,0 & 1 & 100,0 & 10 & 100,0 & 7 & 100,0 & 49 & 80,3 \\
\hline & $\mathrm{N}$ & - & 0,0 & 12 & 30,7 & - & 0,0 & - & 0,0 & - & 0,0 & - & 0,0 & 2 & 19,7 \\
\hline \multirow{2}{*}{ População-alvo } & $S$ & 2 & 100,0 & 37 & 94,8 & 2 & 100,0 & 1 & 100,0 & 10 & 100,0 & 7 & 100,0 & 59 & 96,7 \\
\hline & $\mathrm{N}$ & - & 0,0 & 2 & 5,2 & - & 0,0 & - & 0,0 & - & 0,0 & - & 0,0 & 2 & 3,3 \\
\hline \multirow{2}{*}{ Objetivos } & $S$ & 2 & 100,0 & 22 & 56,4 & 2 & 100,0 & I & 100,0 & 10 & 100,0 & 7 & 100,0 & 44 & 72,1 \\
\hline & $\mathrm{N}$ & - & 0,0 & 17 & 43,6 & - & 0,0 & - & 0,0 & - & 0,0 & - & 0,0 & 17 & 27,9 \\
\hline \multirow{2}{*}{ Proposta } & $S$ & 2 & 100,0 & 9 & 23,0 & 2 & 100,0 & 1 & 100,0 & 10 & 100,0 & 7 & 100,0 & 25 & 40,9 \\
\hline & $\mathrm{N}$ & - & 0,0 & 30 & 77,0 & - & 0,0 & - & 0,0 & - & 0,0 & - & 0,0 & 36 & 59,1 \\
\hline Papel do & $S$ & 1 & 50,0 & 10 & 25,6 & 2 & 100,0 & 1 & 100,0 & 6 & 60,0 & 6 & 85,7 & 25 & 44,2 \\
\hline enfermeiro & $\mathrm{N}$ & - & 50,0 & 29 & 74,4 & - & 0,0 & - & 0,0 & 4 & 40,0 & 1 & 14,3 & 36 & 55,8 \\
\hline
\end{tabular}


se Que só responderam a esta Questão aQueles Que afirmaram sim para a Questão "Conhece o SAMVVIS?", totalizando 55 enfermeiras(os).

\section{DISCUSSÃO}

Neste estudo, observou-se Que a maioria dos profissionais de enfermagem Que compunham o universo é majoritariamente do sexo feminino, confirmando Que nas instituições de saúde, de um modo geral, as enfermeiras são Quantitativamente maior número, pois historicamente as atividades do cuidar sempre foram atribuídas à mulher, de tal modo Que a profissão foi se tornando eminentemente feminina ${ }^{(9)}$.

No tocante as informações sociais, o estudo evidencia Que a maioria são profissionais com pouco tempo de ingresso no serviço e com formação além da graduação, o Que lhes dariam maior possibilidade de conhecimento sobre o SAMVVIS, referência no Estado do Piauí ao atendimento às mulheres vítimas de violência sexual e que funciona na mesma maternidade onde este grupo desenvolve atividades de enfermagem voltadas para a saúde da mulher.

O estudo mostra Que a maioria dos pesquisados conhece o SAMVVIS, mas os percentuais afirmativos vão decrescendo à medida Que respondem sobre o conhecimento do funcionamento, os objetivos, o papel do enfermeiro na equipe e a proposta de organização. A violência não é em si mesmo objeto próprio da área da saúde, mas seu contexto multifacetado e o impacto Que causa nas pessoas exigem atenção e cuidados dos profissionais dessa área ${ }^{(14)}$. Entende-se Que a dimensão desse fenômeno impõe aos serviços de saúde - em especial aqueles cujas atividades são voltadas às mulheres, nos Quais se oferece um serviço especializado à mulher vítima de violência sexual -, Que os profissionais conheçam a proposta, como funcionam e Que estejam todos engajados para ajudar essas mulheres.

É importante salientar Que, no cotidiano do cuidar, os profissionais de enfermagem se defrontam com situações de violência, principalmente voltadas à mulher, devendo, portanto, buscar conhecimentos que os habilitem a acolher, cuidar e promover a prevenção ${ }^{(15)}$. Dada à complexidade da situação e as graves
conseQüências impostas às vítimas, a organização dos serviços deve garantir um atendimento humanizado, integral e de Qualidade às mulheres. Para tal, o ideal é Que o atendimento seja prestado por uma equipe multidisciplinar e Que esteja comprometida com este tipo de atendimento ${ }^{(11)}$.

O estudo também retrata Que foi por intermédio da própria instituição Que a maioria dos sujeitos obteve as informações sobre o SAMVVIS. Embora o serviço funcione na mesma instituição, para alguns pesquisados esse conhecimento foi repassado em cursos, meios de comunicação e outros. A intervenção em situações de violência sexual exige a atuação de profissionais sensibilizados e preparados tecnicamente para prestar uma assistência adequada. Para tanto, faz-se necessário promover a formação de pessoal especializado e capacitado para atender e oferecer suporte às suas principais demandas. A capacitação deve incluir, além de conhecimentos técnicos, reflexões sobre o problema da violência sexual, crenças pessoais e o contato direto com as vítimas de agressão ${ }^{(16)}$.

A Norma Técnica no 6/05 do Ministério da Saúde coloca Que os serviços de saúde devem ter conhecimento sobre os locais de atendimento à mulher vítima de violência sexual e inclusive dispor de lista, Que deve ser de acesso a todos os funcionários do serviço, oportunizando, assim, às mulheres Que estejam em situação de violência ser encaminhadas a estes locais.

\section{CONCLUSÕES}

O estudo possibilitou uma aproximação acerca do conhecimento dos enfermeiros sobre o SAMVIS, evidenciando que os mesmos demonstram saberem sobre o serviço, mas sem envolvimento com o mesmo.

Conclui-se da necessidade de divulgação desse atendimento especializado junto aos enfermeiros da instituição, notadamente nos aspectos dos objetivos, da proposta de organização e do papel do enfermeiro, bem como da política nacional de atenção às mulheres vítimas de violência sexual. Nesse sentido, sugere-se a formação de grupos de discussão e a implantação da consulta de enfermagem no SAMVVIS, o Que contribuirá para fortalecer o apoio à mulher vitimizada.

\section{REFERÊNCIAS}

I. Monteiro CFS, Araújo TME, Nunes BMVT, Lustosa AR, Bezerra $\mathrm{CMI}$. A violência contra a mulher atendida em unidade de urgência: uma contribuição da enfermagem. Esc Anna Nery Rev Enferm 2006; 10(2): 273-9.

2. Campos MAMR. Violência Sexual como uma Questão de Saúde Pública: atenção específica em serviço de saúde [dissertação]. São Paulo (SP): Faculdade de Saúde Pública, Universidade de São Paulo; 2006.

3. Oliveira EM. Violência sexual e saúde. Cad Saúde Pública 2007; 23(2): 455-8.

4. Vilella WV, Lago T. Conquistas e desafios no atendimento das mulheres Que sofreram violência sexual. Cad Saúde Pública 2007; 23(2): 47I-5.

5. Monteiro CFS, Costa NSS, Nascimento PSV, Aguiar YA. Violência intrafamiliar contra adolescentes grávidas. Rev Bras

Enferm 2007; 60(4): 373-6.

6. Drezett I, Caballero M, Juliano Y, Prieto ET, Marques IA, Fernandes CE. Estudo de mecanismos e fatores relacionados com o abuso sexual em crianças e adolescentes do sexo feminino. I Pediatr 2001; 77(5): 413-9.

7. Rotania AA, Dias IMV, Sousa KV, Wolff LR, Reis LB, Tyrrel MAR. Violência contra a mulher: o perigo mora da porta para dentro. Esc Anna Nery R Enferm 2003 7(1): 1 14-25.

8. Drezett J. Estudo de fatores relacionados com a violência sexual contra crianças, adolescentes e mulheres adultas [tese]. São Paulo (SP): Centro de Referência da Saúde da Mulher e de Nutrição, Alimentação e Desenvolvimento Infantil; 2000.

9. Seuinca F, Diniz D, Braga K. Violência sexual contra a mulher: um desafio para o ensino e a pesquisa no Brasil. Rev Bioética 2006; 12(2): 127-35. 
10. Oliveira EM. Atendimento às mulheres vítimas de violência sexual: um estudo qualitativo. Rev Saúde Pública 2005; 39(3): 376-82.

II. Ministério da Saúde (BR). Secretaria de Atenção à Saúde. Departamento de Ações Programáticas Estratégicas. Área Técnica de Saúde da Mulher. Prevenção e Tratamento dos Agravos resultantes da Violência Sexual contra Mulheres e Adolescentes: norma técnica $2^{\text {a }}$ ed. Brasília (DF): Ministério da Saúde; 2005.

12. Secretaria de Estado da Saúde do Piauí (PI). Proposta de organização de serviço para atenção às mulheres vítimas de violência sexual - SAMVVIS. Teresina (PI); 2004.
13. Secretaria de Estado da Saúde do Piauí (PI). Coordenação de Atenção à Saúde da Mulher. Teresina (PI); 2006.

14. Minayo MCS. Violência: um problema para a saúde dos brasileiros. In: Ministério da Saúde (BR). Secretaria de Vigilância em Saúde. Impacto da violência na saúde dos brasileiros. Brasília (DF): Ministério da Saúde; 2005. p. 10-30.

15. Elsen I. A violência bate a porta. In: Luz AMH, Mancia JR, Motta MGC, organizadores. As amarras da violência: a família, as instituições e enfermagem. Brasília (DF): Associação BrasileiradeEnfermagem; 2004. p. 171-5.

16. Meyer DEE. Processos coletivos de produção de conhecimento em saúde: um olhar sobre o exercício de enfermagem no hospital. Rev Bras Enferm 2006; 59(1): 95-9. 